\title{
Modulation de l'expression des fonctions différenciées chez des chondrocytes immortalisés par SV4O
}

\author{
Sophie Thénet, Sylvie Demignot, Monique Adolphe
}

$\mathbf{L}$ e chondrocyte, cellule constitutive du cartilage articulaire, assure le maintien de l'intégrité de ce tissu en synthétisant principalement deux types de macromolécules, marqueurs du phénotype différencié : les protéoglycannes du cartilage et le collagène de type II. Lorsque les chondrocytes sont isolés de la matrice cartilagineuse et placés en culture monocouche, on observe, dans un certain nombre de situations, une transition de la synthèse de ces macromolécules à d'autres types de collagènes et de protéoglycannes génétiquement distincts. Au niveau de la synthèse du collagène, cela se traduit essentiellement par une forte diminution, voire la disparition de la synthèse du collagène de type II, accompagnée de l'apparition de collagènes de types I et/ou III qui sont normalement très faiblement représentés dans le chondrocyte différencié [1]. Par ailleurs, il a été montré que des chondrocytes "dédifférenciés" par des subcultures successives ou après traitement à l'acide rétinoïque pouvaient réverser vers le phénotype différencié après culture en gel d'agarose ou après modification des microfilaments par la dihydrocytochalasine $B \quad[2,3]$. L'identification des mécanismes responsables de la " dédifférenciation " et " rediffé-

S. Thénet, S. Demignot, M. Adolphe : Laboratoire de pharmacologie cellulaire, École pratique des hautes études, Institut biomédical des Cordeliers, 15, rue de l'École-deMédecine, 75006 Paris, France. renciation " in vitro permettrait une meilleure compréhension de la régulation du métabolisme physiologique de cette cellule ainsi que des dérèglements observés dans les situations pathologiques telles que l'arthrose ou les maladies inflammatoires (arthrite rhumatoïde).

L'une des approches pour appréhender ces mécanismes a été l'étude de la modulation de la différenciation du chondrocyte sous l'influence de différents oncogènes rétroviraux (myc, milvaf, src). En outre, l'immortalisation de chondrocytes aviaires et de mammifères par l'oncogène myc a été rapportée dans la littérature $[4,5]$. Dans le cas des chondrocytes de mammifères (rat), la synthèse d'une matrice de protéoglycannes colorable par le bleu Alcian était maintenue. En revanche, la synthèse de collagène de type II était fortement diminuée [5]. La possibilité de réexpression du collagène de type II n'a pas été étudiée.

Immortalisation de chondrocytes articulaires par les fonctions précoces de SV40

La transfection de chondrocytes articulaires de lapin en culture primaire, par un plasmide portant les fonctions précoces de SV40, nous a permis d'isoler plusieurs clones de chondrocytes immortalisés (ayant franchi plus de 130 passages, la durée de vie normale in vitro du chondrocyte articulaire de lapin étant de 8 ou 9 passages). Ces cellules expriment l'antigène grand $T$ de SV40, présentent un caryotype hypotétraploïde et une tumorigénicité faible chez la souris nude [6].

La synthèse de matrice colorable par le bleu Alcian est diminuée d'un facteur environ 20x. L'analyse du collagène synthétisé, par électrophorèse bidimensionnelle après clivage des chaînes de collagène au bromure de cyanogène, révèle que le collagène de type I trimérique est synthétisé de façon majoritaire. Aucune trace de collagène de type II ne peut être détectée. L'analyse des ARN totaux par Northern blot montre que l'inhibition de la synthèse du collagène de type II se situe au niveau transcriptionnel. En revanche, les ARN codant pour la chaîne $\alpha 1(\mathrm{I})$ du collagène de type I peuvent être mis en évidence au moins jusqu'au $80^{\mathrm{e}}$ passage.

Les chondrocytes immortalisés par SV40 ont alors été cultivés dans des conditions qui permettent à des chondrocytes normaux dédifférenciés soit par subculture, soit après traitement à l'acide rétinoïque, de réexprimer le collagène de type II de façon majoritaire : culture tridimensionnelle en gel de collagène ou d'agarose, culture monocouche en présence de dihydrocytochalasine $B$ et de TGF $\beta$. La réexpression de collagène de type II par les chondrocytes immortalisés n'a pu être mise en évidence dans aucune de ces situations.

$\mathrm{m} / \mathrm{s} n^{\circ} 4$ vol. 7 , auril 91 
La transfection de chondrocytes fraîchement isolés du cartilage (avant la mise en culture) avec les gènes précoces de SV40 et la sélection des cellules immortalisées en culture formant des agrégats a conduit à des résultats identiques. La dédifférenciation précoce qui affecte les chondrocytes lors du transfert de leur environnement physiologique tridimensionnel à la culture monocouche n'est donc pas seule responsable de l'incapacité des chondrocytes immortalisés par SV40 à réexprimer le collagène de type II. Le phorbol-12-myristate-13-acétate (PMA), activateur de la protéine kinase $C$, est un puissant agent dédifférenciant des chondrocytes. A l'inverse, les inhibiteurs de la protéine kinase $\mathrm{C}$ comme la staurosporine sont capables de réinduire le phénotype collagène différencié chez le chondrocyte dédifférencié. Mais il semble que l'activation de la protéine kinase $\mathrm{C}$ bloque la réexpression par tous les autres inducteurs ( $\mathrm{P}$. Benya, communication personnelle). L'activation de la protéine kinase $\mathrm{C}$, sous l'influence de l'expression des fonctions précoces de SV40, pourrait être responsable du blocage observé. Cette hypothèse pourra être vérifiée par l'utilisation de la staurosporine. Par ailleurs, la transfection de chondrocytes par un plasmide portant l'antigène $T$ d'un mutant thermosensible de SV40 permettra de contrôler l'expression de ce dernier, de déterminer si une réversion vers le phénotype différencié peut être obtenue à température non permissive ou si l'expression de SV40-T et/ou la culture à long terme entraînent une suppression définitive de ce phénotype

Remerciements

Nous remercions très sincèrement le $\mathrm{Dr} \mathrm{J}$. Feunteun pour son aide lors de l'initiation de ce travail et pour le don des plasmides SV40.

Ce travail a été soutenu par le ministère de la Recherche et de l'Enseignement supérieur (contrat $n^{\circ}$ 85.C.0682) et par l'Inserm (contrat $\left.\mathrm{n}^{\circ} 897001\right)$.

$\mathrm{m} / \mathrm{s} n^{\circ} 4$ vol. 7 , avril 91

\section{Summary}

Modulation of the expression of differentiated functions in SV40 immortalized chondrocytes

Rabbit articular chondrocytes were irnmortalized after transfection with a plasmid coding for SV40 early functions. These cells express SV40 large $T$ antigen and display a hypotetraploid karyotype and a weak tumorigenicity in nude mice. While normal chondrocytes express almost exclusively type II collagen, these cells synthesize type I trimer collagen in majority. Assays for type II collagen reexpression revealed that immortalized chondrocytes were unable to reexpress the differentiated phenotype in a variety of culture conditions which have been shown to make normal dedifferentiated chondrocytes reexpress type II collagen. The possible role of protein kinase $\mathrm{C}$ activation in this phenotype suppression is under investigation. In addition, articular chondrocytes will be transfected with a temperature-sensitive SV40 large $T$ antigen in order to determine if type II collagen can be reexpressed at the nonpermissive temperature.

\section{Références}

1. Benya PD, Padilla SR, Nimni ME. The progeny of rabbit articular chondrocytes synthesize collagen types I and III and type I trimer but not type II. Verification by cyanogen bromide peptide analysis. Biochernistry $1977 ; 16: 865-72$.

2. Benya PD, Shaffer JD. Dedifferentiated chondrocytes reexpress the differentiated collagen phenotype when cultured in agarose gel. Cell $1982 ; 30: 215-24$.
3. Benya PD, Brown PD, Padilla SR. Microfilament modification by dihydrocytochalasin $\mathrm{B}$ causes retinoic acid-modulated chondrocytes to reexpress the differentiated collagen phenotype without a change in cell shape. J Cell Biol $1986 ; 106: 161-70$.

4. Gionti E, Pontarelli G, Cancedda R. Avian myelocytomatosis virus immortalizes differentiated quail chondrocytes. Proc Natl Acad Si USA 1985 ; 82 : 2756-60.

5. Horton WE, Cleveland J, Rapp U, et al. An established rat cell line expressing chondrocytes properties. Exp Cell Res 1988; 457-68.

6. Thénet $\mathrm{S}$, Adolphe $\mathrm{M}$. Immortalization of chondrocytes in culture. In: Maroudas A, Kuettner K, eds. Methods in Cartilage Research. London: Acad Press, 1990 : 95-8. 